University of Missouri-Kansas City School of Law

UMKC School of Law Institutional Repository

\title{
In re: Huping Hu: Quantum Entanglement, Medical Innovation, and Patentability on the Scientific "Fringe".
}

Christopher M. Holman

University of Missouri - Kansas City, School of Law

Follow this and additional works at: https://irlaw.umkc.edu/faculty_works

\section{Recommended Citation}

Christopher M. Holman, In re: Huping Hu: Quantum Entanglement, Medical Innovation, and Patentability on the Scientific "Fringe”., 40 Biotechnology Law Report 222 (2021).

Available at: https://irlaw.umkc.edu/faculty_works/219 
PRE-PRINT VERSION (For print version see Christopher M. Holman, In re Huping Hu:

Quantum Entanglement, Medical Innovation, and Patentability on the Scientific "Fringe," 40

BIOTECHNOLOGY LAW REPORT 222 (2021)).

\title{
In re Huping Hu: Quantum Entanglement, Medical Innovation, and Patentability on the Scientific "Fringe"
}

\author{
Christopher M. Holman*
}

\begin{abstract}
In a recently issued non-precedential decision, In re Huping $\mathrm{Hu}$, the Federal Circuit affirmed the PTO's rejection of patent claims based on the incredible nature, and presumed inoperability, of the claimed invention. The would-be inventors, a husband-and-wife team both having have PhDs from the University of Illinois at ChampaignUrbana, claim to have discovered how to harness "quantum entanglement" to achieve "non-local" manipulation of water, and even more fantastically, manipulation of a human subject that has ingested the water. For example, they assert that they can quantum entangle a sample of water, divide the water into two portions, and have a human test subject drink one of the portions. Later, if someone adds a drug to the other portion, they claim that the drug will act upon the test subject, even if the test subject is thousands of miles away. This article provides a brief introduction to the field of quantum entanglement, and the patenting of practical applications of this "spooky" phenomenon. It then turns to a discussion of patent law's utility requirement, and how it is applied by the PTO during examination of patent applications, particularly with respect to claimed inventions deemed incredible and thus presumptively inoperative. The article then summarizes the prosecution history, and subsequent unsuccessful appeal, of one of the patent applications at issue in $\mathrm{In} \mathrm{re} \mathrm{Hu}$, followed by some concluding thoughts.
\end{abstract}

U.S. patent law's utility requirement denies patentability to any purported invention lacking demonstrable usefulness in a practical, real-world sense. In practice, the utility requirement rarely comes into play with respect to technologies other than chemistry and biology, which patent law has categorized as relatively unpredictable. Most commonly, it comes up in the

\footnotetext{
* Christopher M. Holman, Professor of Law, University of Missouri-Kansas City School of Law; Senior Fellow, Center for the Protection of Intellectual Property, George Mason University, Antonin Scalia Law School; and Executive Editor, Biotechnology Law Report.
} 
context of a purported human therapeutic, with respect to which the patent application as filed must evince a specific, substantial, and credible use in order to satisfy the requirement. In most cases, the issue for the U.S. Patent and Trademark Office (PTO) or court to decide is whether the patent applicant has disclosed a therapeutic use that is both "specific" and "substantial." "The standard for assessing whether a patent application satisfies these requirements is set forth in case law, most significantly In re Brana and its progeny. The PTO's interpretation and implementation of this case law is set forth in Section 2107 of the Manual of Patent Examining Procedure (MPEP).

In the modern era, especially post-Brana, the "credibility" aspect of the test for practical utility has come up much less often, particularly with respect to purported inventions involving human therapeutics. Historically, the PTO played a much more active role in attempting to deny patentability to inoperative medicinal inventions, largely in an attempt to combat the problem of so-called "patent medicines," which were touted to provide tremendous therapeutic benefits to those that ingested them, but which in fact were ineffective at best, and often harmful and dangerous, containing addictive drugs such as morphine, or even toxic agents. Purveyors of these patent medicines often obtained U.S. patents on them, and when marketing the products pointed to these issued patents as supposed evidence that the U.S. government had somehow validated their safety and efficacy. In view of this problem, is not surprising that the PTO sought to protect the public by denying patent protection to ineffective and unsafe purported medicines. ${ }^{2}$

But since the 1960s the U.S. Food and Drug Administration (FDA) has taken over the role of regulating the safety and efficacy of products marketed as human therapeutics, which generally involves evidence including data from human clinical trials. ${ }^{3}$ The In re Brana decision recognized that reality, and essentially directed the PTO to lower the utility bar for purported human therapeutics, and leave the evaluation of safety and efficacy to FDA. Under Brana and its progeny, a patent applicant is not required to prove up therapeutic safety and efficacy in a human in the manner required for FDA approval, and generally human clinical trial data is not a prerequisite to patenting. The standard for satisfying the utility requirement with respect to human therapeutic is more about plausibility, and can often be satisfied with in vivo or animal testing data, and sometimes even that is not required if the applicant can articulate a possible basis for inferring a reasonable likelihood that a compound or treatment will have some use as a human therapeutic. In most cases involving a method for treating a human subject with a

\footnotetext{
${ }^{1}$ In re Brana, 51 F.3d 1560 (Fed. Cir. 1995).

${ }^{2}$ See, e.g., Ex Parte Moore, 128 U.S.P.Q. (BNA) ๆ 8, , 1960 WL 7929 (Dec. 20, 1960)(“Great care and scrutiny should be particularly taken in connection with applications for medical patents. While the granting of a patent does not legally constitute a certificate that the medicine to which it relates is a good medicine and will cure the disease or successfully make the test which it was intended to do, nevertheless, the granting of such a patent gives a kind of official imprimatur to the medicine in question on which as a moral matter some members of the public are likely to rely. In view of these circumstances, it is right and proper that the Patent Office should be very careful and perhaps even reluctant to grant a patent on a new medical formula until it has been thoroughly tested and successfully tried by more than one physician.” Citing Isenstead v. Watson, 157 F. Supp. 7, 8 (D.D.C 1957)).

${ }^{3}$ Michelle Meadows, Promoting Safe \& Effective Drugs for 100 Years, FDA Consumer Magazine-The Centennial Edition (January-February 2006)(discussing the Kefauver-Harris Drug Amendments of 1962), available at https://www.fda.gov/about-fda/histories-product-regulation/promoting-safe-effective-drugs-100-years.
} 
pharmaceutical agent, a submission of experimental test results purporting to demonstrate the plausibility of the asserted therapeutic utility, i.e., data from in vivo or animal testing, will be sufficient. As a general matter, Brana and its progeny direct the PTO to accept such data, unless the PTO has some substantial basis for challenging its plausibility.

In contrast, PTO rejections of therapeutic patent claims, and indeed patent claims in general, for lack of credibility are relatively rare, as specifically noted in the MPEP. ${ }^{4}$ When the PTO finds a patent applicant's asserted utility to be incredible, it will classify the claimed invention as "inoperative," and as a consequence unpatentable under the utility requirement. For example, claims directed towards purported inventions involving a perpetual motion machine, or so-called "cold fusion," have been deemed inoperative, and the Federal Circuit has upheld PTO decisions denying these incredible inventions patent protection. ${ }^{5}$ Perpetual motion machines and cold fusion both violate the fundamental laws of physics, at least as understood by what the PTO considers to be "mainstream" science, and the decision to deny them the implicit imprimatur of a U.S. patent is probably a good thing.

Of course, the potential danger is that truly groundbreaking and revolutionary innovations might be denied patent protection if the PTO perceives the technology to be incredible, and thus presumptively inoperative. In fact, there was a time when the PTO sought to deny patent protection to inventions involving the treatment of cancer, ${ }^{6}$ or restoration of hair growth, ${ }^{7}$ finding these technologies to be incredible and presumptively inoperative. This is understandable, given the history of "patent medicines." The fear would be that a patent on an inoperative cancer cure, or method of restoring hair growth, could be used to persuade a credulous consumer that a U.S. agency had somehow verified the incredible claim. However, later in the $20^{\text {th }}$ century, as we all know, treatments for cancer and medicines for restoring hair growth both became a reality, and this fact was reflected in Federal Circuit decisions instructing the PTO not to reject patent claims directed towards this area of technology based on any inherent presumption of lack of therapeutic utility. ${ }^{8}$

\footnotetext{
${ }^{4}$ MPEP $\$ 2107$.

${ }^{5}$ Newman v. Quigg, F.2d 1575 (Fed. Cir. 1989)(rejection of patent claiming "Energy Generation System Having Higher Energy Output Than Input”); In re Swartz, 50 F. App'x 422 (Fed. Cir. 2002)(rejection of claims directed to "cold fusion").See also, Tol-O-Matic, Inc. v. Proma Product-und Marketing Gesellschaft M.b.H., 945 F.2d 1546, 1552 (Fed. Cir. 1991)(“Section 101 has ... been interpreted to exclude devices deemed to be scientifically impossible, such as perpetual motion machines."; Blacklight Power, Inc. v. Rogan, 295 F.3d 1269 (Fed. Cir. 2002) (PTO withdrew a patent application from issue after a Group Director determined that the claimed invention appeared to be contrary to the "known laws of physics and chemistry.").

${ }^{6}$ Application of Citron, 325 F.2d 248, 252 (C.C.P.A. 1963)(“...the disclosure of the cure of human cancer as a field of utility is incredible and misleading unless proven by statistically significant evidence.");

${ }^{7}$ See, e.g., In re Ferens, 417 F.2d 1072 (CCPA 1969); In re Oberweger, 115 F.2d 826, 829 (CCPA 1940).

${ }^{8}$ In re Jolles, 628 F.2d 1322, 1327 (C.C.P.A. 1980)(“ “...the medical treatment of a specific cancer is not such an inherently unbelievable undertaking or involves such implausible scientific principles as to be considered incredible."); Ex parte Rubin, 5 USPQ2d 1461, 1462 (Bd. Pat. App. \& Int'f 1987) (“The contemporary knowledge in the art has far advanced since the days when any statement of utility in treating cancer was per se incredible."); In re Cortright, 165 F.3d 1353, 1357 (Fed. Cir. 1999) ("Treating baldness was once considered an inherently unbelievable undertaking. Since then, however, treatments for baldness have gained acceptance. Rogaine ${ }^{\circledR}$ (minoxidil) and Propecia ${ }^{\circledR}$ are recognized as effective in treating baldness. In addition, the PTO has granted approximately one hundred patents on methods of treating baldness.").
} 
In a recently issued non-precedential decision, In re Huping Hu, the Federal Circuit affirmed the PTO's rejection of patent claims directed towards, inter alia, methods of delivering medication or anesthesia to a human subject, based on the incredible nature of the claimed invention, and a presumption of inoperability. ${ }^{9}$ On its face, it might seem that the applicants had made a good case for the operability of their purported invention. A husband-and-wife team, the purported inventors both have PhDs from the University of Illinois at Champaign Urbana. The husband (Huping $\mathrm{Hu}$ ) has a $\mathrm{PhD}$ in biophysics; I was unable to ascertain the subject matter of his wife's (Maoxin Wu). Hu also has a JD from New York Law School, and his own legal practice in New York. Wu has an MD from China, and is a clinical professor at Stony Brook University's medical school. The patent applications include numerous working examples providing data from "verification experiments" purporting to demonstrate the operability of the claimed inventions. This data also appears in a number of scientific articles authored by the purported coinventors and published in peer-reviewed journals. Prior to the final rejection of the claims by the PTO, related claims were allowed to issue in patents in the United Kingdom and China. In view of the backgrounds of the applicants, the working examples in the application as filed, the publications and non-U.S. patents, one would think that the applicants had met their burden to overcome any presumption of inoperability.

The answer, however, lies in the nature of the claimed inventions, which will strike most people as incredible indeed. To summarize, $\mathrm{Hu}$ and $\mathrm{Wu}$ (hereafter "Hu") claim to have discovered how to harness "quantum entanglement" to achieve "non-local" manipulation of water, and even more fantastically, manipulation of a human subject that has ingested the water. For example, they assert that a sample of water (typically a volume of several hundred milliliters) can be quantum entangled by irradiating the water using, for example, a microwave oven, a laser light, or a magnetic pulse. Once the sample of water has become entangled, it can be divided into two (or presumably more) portions, and the two portions will remain quantum entangled, and effectively coupled. As a consequence of this coupling, actions taken on one portion will be experienced by the other. If the temperature of one portion is changed, a corresponding change will be observed in the other portion. Likewise, if the $\mathrm{pH}$ of one portion is altered, a corresponding alteration will occur in the other portion. According to the inventors, this will occur no matter how far apart the two portions are - in some of their experiments, the separation is thousands of miles (i.e., the distance from New York to Beijing).

The medical treatment aspects of their purported invention are even more incredible. They assert in their patent applications that they can quantum entangle a sample of water (using, for example, a microwave oven), divide the water into two portions, and have a human test subject drink one of the portions. Later (after 30 minutes, for example) someone adds a medication (e.g., ephedrine, morphine, oxycodone, nicotine, or caffeine) or an anesthetic (chloroform or diethyl ether) to the other portion, and the added medication or anesthetic will act upon the test subject, even if the test subject is thousands of miles away from the added medication or anesthetic. In their "verification testing," the test subject did not know if a substance was added to the remotely located portion of water, or what that substance was. Negative controls were

\footnotetext{
${ }^{9}$ In re Huping Hu, No. 2019-2104, 2021 WL 1016437 (Fed. Cir. Mar. 17, 2021).
} 
used in which only water was added. Nevertheless, the test subject reported feeling affects consistent with how a person would feel after being exposed to the specific medication or anesthetic added to the other portion of water. In the case of ephedrine, a heart stimulant, the researchers measured an elevated heart rate after addition of ephedrine (in the form of the overthe-counter medication Primatene) to the "non-local" water. The patent applications include numerous working examples providing data from multiple trials involving different medications and anesthetics, that on their face purport to confirm the incredible results claimed in the patent application.

This article provides a brief introduction to the field of quantum entanglement, and the patenting of practical applications of this "spooky" phenomenon. It then turns to a discussion of patent law's utility requirement, and how it is applied by the PTO during examination of patent applications, particularly with respect to claimed inventions deemed incredible and thus presumptively inoperative. The article then summarizes the prosecution history, and subsequent unsuccessful appeal, of one of the patent applications at issue in In re $\mathrm{Hu}$, followed by some concluding thoughts.

\section{Quantum entanglement}

My background is in chemistry and molecular biology, not physics, and prior to reading In re $\mathrm{Hu}$ I was unfamiliar with the concept of quantum entanglement. But I looked into it, and found that it is in fact a well-substantiated and fascinating manifestation of quantum mechanics that is currently the subject of a great deal of interest. I believe I have gained some appreciation for the subject, and perhaps some understanding, at least at the very high level of abstraction. That said, I cannot claim to understand it, but in that I am surely not alone. Nobel laureate Richard Feynman has been credited with the observation that "if you think you understand quantum mechanics, you don't understand quantum mechanics." Nonetheless, I will attempt to provide an introduction to quantum entanglement for those unfamiliar with the topic, so as to better understand the invention claimed by $\mathrm{Hu}$, and more importantly, the potential for more credible applications of quantum entanglement to medicine and biotechnology that might someday actually come to pass.

To try to get my head around the bizarre world of quantum mechanics and quantum entanglement, I turned to YouTube, where I found a plethora of videos addressing the subject in layman's terms. One that I found particularly helpful in this regard is entitled "Quantum Entanglement: Spooky Action at a Distance," presented by Dr. Don Lincoln on his YouTube channel Fermilab. ${ }^{10}$ Dr. Lincoln conducts research in particle physics at Fermi National Accelerator Laboratory, and is an adjunct professor of physics at the University of Notre Dame. Through his Fermilab YouTube channel he communicates principles of physics to laypeople. The following simplified introduction to quantum entanglement relies heavily on his video. I think I am accurately conveying the essence of Dr. Lincoln's explanation, but to the extent I

\footnotetext{
${ }^{10}$ Fermilab, Quantum Entanglement: Spooky Action at a Distance (posted to YouTube on February 12, 2020), available at https://www.youtube.com/watch? $\mathrm{v}=\mathrm{JFozGfxmi} 8 \mathrm{~A} \& \mathrm{t}=8 \mathrm{~s}$.
} 
might fall short, I apologize, and take full responsibility. Dr. Lincoln is an educator who clearly seems to know his business.

In his video, Dr. Lincoln describes quantum entanglement, a manifestation of quantum mechanics, as the "weirdest physics phenomenon" he has ever learned about. Quantum mechanics, and more specifically quantum entanglement, is usually associated with very small objects, e.g., subatomic particles, atoms and molecules, but more recently physicists have come to believe that it also applies to macroscopic objects, including, as Dr. Lincoln notes, human beings.

Nonetheless, quantum entanglement is more readily explained (and observed) in terms of subatomic particles, such as photons or electrons. Quantum mechanics teaches us that the behavior of a subatomic particle is described by a wave function, and that the wave function governs the probability that one will observe the particle in a particular configuration. Prior to measuring the configuration of a subatomic particle, that configuration is indeterminate, existing only as a probability. For example, if the configuration of interest is the direction of spin, a subatomic particle's wave function describes the probability that the spin will be pointing in one direction or the other, i.e., either right or left. The most popular understanding of quantum mechanics is that, prior to measurement, the spin direction is simultaneously in all directions allowed by the wave function. However, when the spin direction is measured the wave function collapses, with the spin becoming fixed in a particular orientation.

It turns out that it is possible to prepare two subatomic particles that are both described by a single wave function, which physicists refer to as entanglement of the particles. This can be accomplished, for example, by taking a parent particle having zero spin and allowing it to decay into two daughter particles. The spin of the parent particle is a conserved quantity, and as a result the two daughter particles must have opposite spin, e.g., if one has a spin pointing to the right, the other must have a spin pointing to the left. This is a simple example of entangled particles, i.e., two particles having opposite spin and sharing the same wave function.

Now here is where things start to get interesting (and indeed spooky). It turns out that the two particles can be separated, by feet, miles, or in principle even light-years, and as long as they don't interact with anything, they will remain entangled. As previously mentioned, quantum mechanics is inherently probabilistic, and prior to measurement both particles are simultaneously in all spin states allowed by the wave function, at a probability governed by the wave function. For example, if the spin direction is measured on the horizontal axis, there could be a $50 \%$ probability that the spin is pointing to the right, and a $50 \%$ probability that the spin is pointing to the left. If the spin of one of the particles is measured in the horizontal direction, the measured spin will be either right or left, each $50 \%$ of the time. If we measure the horizontal direction of one entangled particle, and find that it is pointed to the left, it turns out that the other entangled particle will be pointing to the right $100 \%$ of the time, regardless of how far apart the two particles are located.

The implications of this are entirely counterintuitive, and difficult for most of us to grasp, based on our experience in a macroworld governed by the laws of classical mechanics. Somehow, the 
information that one particle has been measured and found to be pointing in a certain direction is transmitted, instantaneously, to the other particle. This would be impossible in the realm of classical mechanics, where information should not be able to travel from one point to another at a speed faster than light. Nonetheless, the phenomenon has been confimed experimentally. Dr. Lincoln provides a concrete example wherein two entangled particles are separated by a distance of 10 feet, the spin of one particle is measured, and then the spin of the second particle is measured five billionths of a second later. Light travels 1 foot in a billionth of a second, so it would require 10 billionths of a second for information communicated at the speed of light to reach the second particle. Nonetheless, the spin of the second particle will always be found to be pointing in the opposite direction of the first particle, proving that the fact that the spin of the first particle was measured has somehow been conveyed to the second particle faster than the speed of light. In other words, quantum information can travel faster than the speed of light. In 1935 Einstein referred to this transfer of quantum information as "spooky action at a distance," and is part of the explanation for Einstein's reported dislike of quantum mechanics.

One of the reasons quantum entanglement is so intriguing is because of the possibility for its practical application. For example, the use of quantum entanglement in computing, communication, cryptography, radar, and microscopy are all active areas of research and development. Some envision using quantum entanglement to synchronize clocks located around the world. Switzerland reportedly used quantum entanglement in an effort to secure electronic voting from hacking or accidental data corruption. ${ }^{11}$ There is something called an entanglementenhanced microscope, and quantum teleportation is a topic of serious discussion (at least for subatomic particles - we are apparently along ways from the "transporter" envisioned by the creators of Star Trek). If we can learn to harness the power of quantum entanglement, it could revolutionize virtually every aspect of human existence.

Although quantum mechanics has traditionally been thought of as restricted to sub-microscopic particles, i.e., molecules, atoms, and subatomic particles, a 2011 article in Scientific American authored by Vlatko Vedral explains that physicists have increasingly come to believe that it applies to everything, irrespective of size. ${ }^{12}$ The article describes recent experiments demonstrating quantum effects in a growing number of macroscopic systems, and observe that "[t]he quintessential quantum effect, entanglement, can occur in large systems as well as warm ones-including living organisms-even though molecular jiggling might be expected to disrupt entanglement." More recently, there have multiple been reports of quantum entanglement at a macroscopic scale, including in living cells. ${ }^{13}$

\footnotetext{
${ }^{11}$ Paul Marks, Quantum cryptography to protect Swiss election TECHNOLOGY 15 October 2007, available at https://www.newscientist.com/article/dn12786-quantum-cryptography-to-protect-swiss-election/\#ixzz6wjDJZlnj. 12 Vlatko Vedral, Living in a Quantum World, Scientific American (2011).

${ }^{13}$ See, e.g., Bohr Institute, Quantum entanglement realized between distant large objects, September 28, 2020, available at https://phys.org/news/2020-09-quantum-entanglement-distant-large.html; Thomas, R.A., Parniak, M., Østfeldt, C. et al. Entanglement between distant macroscopic mechanical and spin systems. Nat. Phys. 17, 228-233 (2021). https://doi.org/10.1038/s41567-020-1031-5; Jonathan O'Callaghan, "Schrödinger's Bacterium" Could Be a Quantum Biology Milestone, Scientific American (Oct 29, 2018); C Marletto et al, Entanglement between living bacteria and quantized light witnessed by Rabi splitting, J. Phys. Commun. 2 (2018) 101001; Krisnanda, T.,
} 


\section{U.S. patents on applications of quantum entanglement}

The PTO has already begun issuing patents directed towards technologies involving quantum entanglement. A search of the PTO's US Patent \& Trademark Office, Patent Full Text and Image Database, conducted on June 5, 2021, found that the words "quantum entanglement" and/or "quantum entangled" appear in the title of 22 issued U.S. patents, in the abstract of 37 issued patents, and in the claims of 41 issued patents. ${ }^{14}$ A cursory review of some of these patents indicates that they are directed not only towards technologies for studying quantum entanglement, but also practical applications of quantum entanglement in computing, communications, and cryptography. Many of the patents are assigned to well-known corporations and research institutions, such as Boeing, Bank of America, Experian, and the Korea Institute of Science and Technology. Here are a few examples:

United States Patent 10,756,891, issued August 25, 2020 and assigned to The Boeing Company, discloses and claims methods and systems for securely transmitting data using quantum entanglement.

United States Patent 10,997,521, issued May 4, 2021 and assigned to Bank of America Corporation, discloses and claims a method for operating a quantumresilient server-cluster that employs quantum entanglement to verify communication security.

United States Patent 10,735,183, issued August 4, 2020 and assigned to Experian Information Solutions, Inc., discloses a secure method of symmetric encryption for private "smart contacts" among multiple parties in a private peer-to-peer network, which in some implementations would employ quantum entanglement.

United States Patent 9,722,785, issued August 1, 2017 and assigned to Korea Institute of Science and Technology, discloses and claims an apparatus for quantum cryptographic communication that employs quantum entanglement.

\section{The utility requirement and the PTO's treatment of seemingly incredible}

\section{inventions}

Patent law's utility requirement is generally understood to be grounded in two sections of the U.S. Patent Act, 35 U.S.C. 101, which requires that an invention be "useful," and 35 U.S.C. 112(a) (or pre-AIA 35 U.S.C. 112, first paragraph), which requires a patent application to enable the "use" of the invention. In most cases, the utility requirement is not an issue in patent examination. Human therapeutics is the one important area of technology where the utility requirement often presents a significant issue of patentability.

The MPEP instructs its examiners not to reject a patent claim for lack of utility under circumstances in which it is readily apparent that the claimed invention has a well-established

\footnotetext{
Marletto, C., Vedral, V. et al. Probing quantum features of photosynthetic organisms. npj Quantum Inf 4, 60 (2018). https://doi.org/10.1038/s41534-018-0110-2.

${ }^{14}$ US Patent \& Trademark Office, Patent Full Text and Image Database, available at https://patft.uspto.gov/netahtml/PTO/index.html.
} 
utility, i.e., "if (i) a person of ordinary skill in the art would immediately appreciate why the invention is useful based on the characteristics of the invention (e.g., properties or applications of a product or process), and (ii) the utility is specific, substantial, and credible." 15

The MPEP explains:

Deficiencies under the "useful invention" requirement of 35 U.S.C. 101 will arise in one of two forms. The first is where it is not apparent why the invention is "useful." This can occur when an applicant fails to identify any specific and substantial utility for the invention or fails to disclose enough information about the invention to make its usefulness immediately apparent to those familiar with the technological field of the invention. Brenner v. Manson, 383 U.S. 519, 148 USPQ 689 (1966); In re Fisher, 421 F.3d 1365, 76 USPQ2d 1225 (Fed. Cir. 2005); In re Ziegler, 992 F.2d 1197, 26 USPQ2d 1600 (Fed. Cir. 1993). The second type of deficiency arises in the rare instance where an assertion of specific and substantial utility for the invention made by an applicant is not credible. ${ }^{16}$

When a PTO examiner rejects a patent claim for lack of utility, it is usually based on a finding that the applicant has failed to assert a specific and substantial utility for the invention. In those rare cases in which an examiner doubts the credibility of an assertion of utility, the examiner will conclude that the claimed invention is wholly ""inoperative" (i.e., it does not operate to produce the results claimed by the patent applicant), and place the burden upon the applicant to prove operability of the invention.

\section{The MPEP notes that:}

Situations where an invention is found to be "inoperative" and therefore lacking in utility are rare, and rejections maintained solely on this ground by a federal court even rarer. In many of these cases, the utility asserted by the applicant was thought to be "incredible in the light of the knowledge of the art, or factually misleading" when initially considered by the Office. In re Citron, 325 F.2d 248, 253 (CCPA 1963). Other cases suggest that on initial evaluation, the Office considered the asserted utility to be inconsistent with known scientific principles or "speculative at best" as to whether attributes of the invention necessary to impart the asserted utility were actually present in the invention. In re Sichert, 566 F.2d 1154, 196 USPQ 209 (CCPA 1977). However cast, the underlying finding by the court in these cases was that, based on the factual record of the case, it was clear that the invention could not and did not work as the inventor claimed it did. Indeed, the use of many labels to describe a single problem (e.g., a false assertion regarding utility) has led to some of the confusion that exists today with regard to a rejection based on the "utility" requirement. Examples of such cases include: an invention asserted to change the taste of food using a magnetic field (Fregeau v. Mossinghoff, 776 F.2d 1034, 227 USPQ 848 (Fed. Cir. 1985)), a perpetual motion machine (Newman v. Quigg, 877 F.2d 1575, 11 USPQ2d 1340 (Fed. Cir. 1989)), a flying machine operating on "flapping or flutter function" (In re Houghton, 433 F.2d 820, 167

\footnotetext{
${ }^{15}$ MPEP $\S 2107$.
}

${ }^{16} I d$. 
USPQ 687 (CCPA 1970)), a "cold fusion" process for producing energy (In re Swartz, 232 F.3d 862, 56 USPQ2d 1703 (Fed. Cir. 2000)), a method for increasing the energy output of fossil fuels upon combustion through exposure to a magnetic field (In re Ruskin, 354 F.2d 395, 148 USPQ 221 (CCPA 1966)), uncharacterized compositions for curing a wide array of cancers (In re Citron, 325 F.2d 248, 139 USPQ 516 (CCPA 1963)), and a method of controlling the aging process (In re Eltgroth, 419 F.2d 918, 164 USPQ 221 (CCPA 1970)). These examples are fact specific and should not be applied as a per se rule. Thus, in view of the rare nature of such cases, Office personnel should not label an asserted utility "incredible," "speculative" or otherwise unless it is clear that a rejection based on "lack of utility" is proper.

The MPEP explains that "[i]n most cases, an applicant's assertion of utility creates a presumption of utility that will be sufficient to satisfy the utility requirement." ${ }^{17}$ In deciding the issue, the PTO applies the Langer test, based on In re Langer and its progeny. ${ }^{18}$

In In re Langer, the Court of Customs and Patent Appeals explained:

As a matter of Patent Office practice, a specification which contains a disclosure of utility which corresponds in scope to the subject matter sought to be patented must be taken as sufficient to satisfy the utility requirement of $\S 101$ for the entire claimed subject matter unless there is a reason for one skilled in the art to question the objective truth of the statement of utility or its scope. ${ }^{19}$

In In re Brana, the Federal Circuit explicitly adopted the Court of Customs and Patent Appeals formulation of the "Langer" standard for 35 U.S.C. 112(a) or pre-AIA 35 U.S.C. 112, first paragraph rejections, as it was expressed in a slightly reworded format in In re Marzocchi, 439 F.2d 220, 223 (CCPA 1971), namely:

[A] specification disclosure which contains a teaching of the manner and process of making and using the invention in terms which correspond in scope to those used in describing and defining the subject matter sought to be patented must be taken as in compliance with the enabling requirement of the first paragraph of $\S$ 112 unless there is reason to doubt the objective truth of the statements contained therein which must be relied on for enabling support. (emphasis added). ${ }^{20}$

Thus, Langer and subsequent cases direct the Office to presume that a statement of utility made by an applicant is true.

The MPEP explains that:

For obvious reasons of efficiency and in deference to an applicant's understanding of the invention, when a statement of utility is evaluated, Office personnel should not begin by questioning the truth of the statement of utility. Instead, any inquiry

\footnotetext{
${ }^{17}$ See, e.g., In re Jolles, 628 F.2d 1322 (CCPA 1980); In re Irons, 340 F.2d 974 (CCPA 1965); In re Langer, 503 F.2d 1380 (CCPA 1974); In re Sichert, 566 F.2d 1154, 1159 (CCPA 1977).

${ }^{18}$ In re Langer, 503 F.2d at 1391.

${ }^{19} \mathrm{Id}$.

${ }^{20} 51$ F.3d 1560 (Fed. Cir. 1995).
} 


\section{PRE-PRINT VERSION}

must start by asking if there is any reason to question the truth of the statement of utility. This can be done by simply evaluating the logic of the statements made, taking into consideration any evidence cited by the applicant. If the asserted utility is credible (i.e., believable based on the record or the nature of the invention), a rejection based on "lack of utility" is not appropriate. Clearly, Office personnel should not begin an evaluation of utility by assuming that an asserted utility is likely to be false, based on the technical field of the invention or for other general reasons.

The MPEP goes on to explain that:

To overcome the presumption of truth that an assertion of utility by the applicant enjoys, Office personnel must establish that it is more likely than not that one of ordinary skill in the art would doubt (i.e., "question") the truth of the statement of utility. ... To do this, Office personnel must provide evidence sufficient to show that the statement of asserted utility would be considered "false" by a person of ordinary skill in the art. Of course, a person of ordinary skill must have the benefit of both facts and reasoning in order to assess the truth of a statement. This means that if the applicant has presented facts that support the reasoning used in asserting a utility, Office personnel must present countervailing facts and reasoning sufficient to establish that a person of ordinary skill would not believe the applicant's assertion of utility.

The MPEP goes on to instruct:

Where an applicant has specifically asserted that an invention has a particular utility, that assertion cannot simply be dismissed by Office personnel as being "wrong," even when there may be reason to believe that the assertion is not entirely accurate. Rather, Office personnel must determine if the assertion of utility is credible (i.e., whether the assertion of utility is believable to a person of ordinary skill in the art based on the totality of evidence and reasoning provided). An assertion is credible unless (A) the logic underlying the assertion is seriously flawed, or (B) the facts upon which the assertion is based are inconsistent with the logic underlying the assertion. Credibility as used in this context refers to the reliability of the statement based on the logic and facts that are offered by the applicant to support the assertion of utility.

One situation where an assertion of utility would not be considered credible is where a person of ordinary skill would consider the assertion to be "incredible in view of contemporary knowledge" and where nothing offered by the applicant would counter what contemporary knowledge might otherwise suggest. Office personnel should be careful, however, not to label certain types of inventions as "incredible" or "speculative" as such labels do not provide the correct focus for the evaluation of an assertion of utility. "Incredible utility" is a conclusion, not a starting point for analysis under 35 U.S.C. 101. A conclusion that an asserted utility is incredible can be reached only after the Office has evaluated both the assertion of the applicant regarding utility and any evidentiary basis of that assertion. The Office should be particularly careful not to start with a presumption that an asserted 
utility is, per se, "incredible" and then proceed to base a rejection under 35 U.S.C. 101 on that presumption.

Rejections under 35 U.S.C. 101 based on a lack of credible utility have been sustained by federal courts when, for example, the applicant failed to disclose any utility for the invention or asserted a utility that could only be true if it violated a scientific principle, such as the second law of thermodynamics, or a law of nature, or was wholly inconsistent with contemporary knowledge in the art. In re Gazave, 379 F.2d 973, 978, 154 USPQ 92, 96 (CCPA 1967). Special care should be taken when assessing the credibility of an asserted therapeutic utility for a claimed invention. In such cases, a previous lack of success in treating a disease or condition, or the absence of a proven animal model for testing the effectiveness of drugs for treating a disorder in humans, should not, standing alone, serve as a basis for challenging the asserted utility under 35 U.S.C. $101 .{ }^{21}$

\section{The prosecution history of a Hu patent application}

This section of the article summarizes the prosecution history of one of the four patent applications at issue in In re Hu. But first, a little on the background of the two applicants, Huping $\mathrm{Hu}$ and his wife Maoxin $\mathrm{Wu}$, who claim to have "harnessed and developed quantum entanglement and nonlocal effects into useful technologies to serve the mankind in many areas, such as communication, engineering, health, medicine and recreation." Note that all of this information has been garnered from webpages associated with $\mathrm{Hu}$ and $\mathrm{Wu}$, and have not been independently validated.

Huping $\mathrm{Hu}$ is originally from China. ${ }^{22}$ He came to the U.S. in 1987, and in 1991 he obtained a $\mathrm{PhD}$ in biophysics from the University of Illinois at Urbana-Champaign. He then went on to obtain his JD from New York Law School in 1998. He is the chief scientist and legal counsel of Biophysics Consulting Group, president of a company called QuantumDream, Inc., and principle of the Law Offices of Huping Hu. "He is also the founder of Scientific God Institute aimed at scientific inquiry on God and Science Association for the New Millennium aimed at a new paradigm of science." 23

Maoxin Wu, also originally from China, holds an MD degree from Shanxi Medical College (P.R. China) and a PhD from the University of Illinois at Urbana-Champaign. ${ }^{24}$ She is board certified in Anatomic and Clinical Pathology and Cytopathology. She is also a Clinical Professor in the Department of Pathology, Stony Brook Medicine, as well as Chief of Cytopathology and Director of Fine Needle Aspiration (FNA) and Core Biopsy Services.

The couple have published numerous articles together, many of which appear to be directed generally to the same general area of technology as the patent applications at issue in In re Hu.

\footnotetext{
${ }^{21}$ MPEP $§ 2107$.

${ }^{22}$ Brief Biography of Huping Hu, NeuroQuantology 2006; 1:4, available at https://neuroquantology.com/datacms/articles/20191022042130pm84.pdf.

${ }^{23} I d$.

${ }^{24}$ Faculty profile for Maoxin $\mathrm{Wu}, \mathrm{MD}, \mathrm{PhD}$, Renaissance School of Medicine, Stony Brook University, available at https://renaissance.stonybrookmedicine.edu/pathology/faculty/wu (last visited June 3, 2021).
} 
Huping $\mathrm{Hu}$ also appears in videos on YouTube where he is apparently presenting his work in this field to colleagues at research institutions in the Czech Republic and Romania. ${ }^{25}$

In re $\mathrm{Hu}$ was a consolidated appeal involving four applications that were not all in the same art unit, and were which processed by two different examiners. For the purposes of this article, I will focus primarily on the prosecution history of one of these applications, U.S. Patent Application No. 13/492,830) ("the '830 application," filed June 9, 2012). The '830 application is titled "Method and Apparatus for Producing and Detecting Non-Local Effects of Substances," and, like the other applications on appeal, recites a "method for communicating between two remote locations through two parts of a quantum-entangled medium with one part being applied to a responsive target such as a particular biological, chemical or other system at one location and a second part being subsequently entangled with a particular substance representing a particular message through quantum-entangling members such as photons at a remote location of arbitrary distance." In particular, the ' 830 application describes non-local effects of medications and anasthetics. The ' 830 application, which published in 2012 as published patent application No. 2012/0253168, claims priority to a provisional application filed in 2006.

For those (like myself) who believe "the name of the game is the claim," and like to see the actual claim language at issue, here are some relevant claims from one of the Hu's applications, U.S. Patent Application No. 13/492,830, as filed:

5. A method of producing and detecting a quantum entanglement between a first target and a second target, and a first non-local effect of said second target on said first target through said quantum entanglement and/or a second non-local effect of said first target on said second target through said quantum entanglement, which comprises the steps of: selecting said first target which comprises a first substance, mixture of substances, physical system, chemical system or biological system; selecting said second target which comprises a second substance, mixture of substances, physical system, chemical system or biological system; providing a first medium at said first location and a second medium at said second location, said first medium being quantum-entangled with said second medium; providing a detecting mean for detecting said quantum entanglement, said first non-local effect and/or said second non-local effect when said mechanism operates; causing said first target to interact with said first medium through a first contact or radiation from a first source; causing said second target to interact with said second medium through a second contact or radiation from a second source; and detecting said quantum entanglement, said first non-local effect and/or said second non-local effect; whereby said quantum entanglement between said first target and said second target, and said first non-local effect through said quantum entanglements and/or said second non-local effect through said quantum entanglements are generated and detected, said non-local effects being physical or chemical, or biological in a human or animal.

${ }^{25}$ See, e.g., https://quantumbrain.org/ for a list of publications and link to recordings of presentations. 


\section{PRE-PRINT VERSION}

9. A method as in claim 5 for sending an encoded message from said first or second location and receiving said encoded message at said second or first location further comprise the steps of: encoding said message to be sent through said second or first non-local effect to said second or first location; and decoding said message received through said second or first non-local effect at said second or first location; whereby said encoded message from said first or second location to said second or first location is sent and received.

10. A method as in claim 9 wherein said first target comprises a first chemical substance, human or animal; said second target comprises a second chemical substance, human or animal; and said first and second media comprise a waterbased medium.

11. A method as in claim 10 wherein said first target comprises an anesthetic, recreational, communicational, or brain or heart stimulating substance; said second target comprises said second human or animal; said first and second sources comprise a magnetic coil connected to a driving mechanism, laser or microwave device; and said detecting mean comprises a first-person experience and report of said experience and/or a detecting device.

12. A method as in claim 11 wherein said first target comprises chloroform, isoflurance, dymethyl ether, ethanol, tribromoethanol, morphine sulfate, fentanyl, nicotine, caffeine or ephedrine; said first contact comprises mixing said first target with said first medium; said second contact comprises delivering orally or intravenously said second medium to said second human or animal; and said detecting device comprising a heart rate monitor, blood pressure monitor, EEG machine or MEG machine.

On appeal, the Federal Circuit only considered one of the patent claims in the ' 830 Application, deeming it representative - claim 5, which had been amended during prosecution:

5. A method of producing and detecting a second plurality of quantum entanglements between a third plurality of quantum entities in a first target and a fourth plurality of quantum entities in a second target, a first non-local effect of said second target on said first target through said second plurality of quantum entanglements and/or a second nonlocal effect of said first target on said second target through said second plurality of quantum entanglements which comprises the steps of:

selecting said first target which comprises a first chemical substance, human or animal at a first location;

selecting said second target which comprises a second chemical substance, human or animal at a second location;

providing a first water-based medium at said first location and a second water-based medium at said second location, a first plurality of quantum entities in said first medium being in a first plurality of quantum entanglements with a second plurality of quantum entities in said second medium; 
providing a detecting means for detecting said second plurality of quantum entanglements, said first non-local effect and/or said second non-local effect when said detecting means operates;

causing said first target to interact with said first water-based medium through a first contact or radiation from a first photon or magnetic pulse generating source;

causing said second target to interact with said second water-based medium through a second contact or radiation from a second photon or magnetic pulse generating source; and

detecting said second plurality of quantum entanglements, said first nonlocal effect and/or said second non-local effect;

whereby said second plurality of quantum entanglements between said third plurality of quantum entities in said first target and said fourth plurality of quantum entities in said second target is generated through said interaction between said third plurality of quantum entities in said first target and said first plurality of quantum entities in said first water-based medium and said interaction between said fourth plurality of quantum entities in said second target and said second plurality of quantum entities in said second water-based medium, and detected through said detecting means; and said first non-local effect of said second target on said first target, comprising a first effect of said second target on a first physical, chemical or biological property or process of said first target, and/or said second non-local effect of said first target on said second target, comprising a second effect of said first target on a second physical, chemical or biological property or process of said second target, are generated through said second plurality of quantum entanglements between said third plurality of quantum entities in said first target and said fourth plurality of quantum entities in said second target and detected through said detecting means.

The patent application includes a number of working examples purporting to verify the efficacy of the use of quantum entanglement to deliver a therapeutic or anesthesia to a remote human subject.

In some of the quantum entanglement "verification experiments," an amount of water was exposed to the radiation of a microwave oven, which presumably was deemed sufficient to quantum entangle the water. A human test subject would then consume half of the water sample, and after 30 minutes from the time of consumption a medication or anesthetic was added to the other half of the sample of microwaved water in a different remote location, and the water was then exposed to laser light or magnetic pulses. In some experiments, the drug or medication was added to the other half of the microwaved water in a room about 50 feet away from the test subject, in other experiments the separation was 50 miles, and in at least one experiment the test subject was over 6000 miles away (in Beijing). The test subject would then be monitored to see if there was any effect from the addition of the medication or anesthetic to the water. 
For example, in some experiments the medication was Primatene, an over-the-counter medication that contains the heart stimulant ephedrine. In these experiments, the heart rate of the test subject was monitored. The applicants reported that additional Primatene to the remote water caused the heart rate of the test subject to significantly increase. In contrast, control experiments in which no Primatene was added to the water did not result in an increase in heart rate.

Other medications that were used in experiments included morphine, fentanyl, oxycodone, nicotine and caffeine. The applicants reported that all of these drugs produced clear and completely reproducible biological and/or chemical effects such as brain effects including euphoria and/or hastened alertness. Anesthetics including chloroform and diethyl ether were also tested and found to be effectively delivered from a remote location via quantum entanglement. The applicants reported that all medications were either leftover items originally prescribed to one of the test subject's late mother, or items available over the counter, and that the general anesthetics used in the study were all "properly obtained for research purposes."

The specification states that "clear and consistently reproducible biological and/or chemical effects such as brain effects were experienced by the test subjects above and beyond what were noticeable in the control portions of the experiments under blind settings," and that "the only possible explanation for the brain effects and other biological and/or chemical effects experienced by the test subjects are that these effects were the consequences of quantum entanglement because the water consumed by the test subjects was never directly exposed to the magnetic pulses or the laser lights in the presence of the substances under studies."

In the first office action issued by the PTO, dated February 11, 2015, the examiner rejected the claims for failure to satisfy the enablement and utility requirements. In assessing the enablement of the claims, the examiner applied the Wands factors, and found, inter alia, the nature of the invention to be "[apparently] drawn to theoretical physics rather than concrete and definable methods." As to the state of the prior art, the examiner found that:

there are two types of prior art in this field. The first is established physical laws and theories and the second is alternative and New Age interdisciplinary physics and consciousness journals. The disclosure of applicant's invention is not found in the established prior art of the first type and Applicant admits that the scientific community is skeptical of the ideas and methods presented herein [citing to statements in the applicant specification, as well as published articles by the applicants]. The teachings of the prior art relevant to the invention are written by the inventor and published in alternative physics journals associated with the inventor.

With respect to the "level of one of ordinary skill in the art" factor, the examiner found that "the disclosure and claims go against established scientific principles and do not describe the invention in a manner which would enable one of ordinary skill in the art (familiar with established physics) to carry out the invention."

With respect to the "level of predictability in the art," the examiner found that "the field of physics has well-established understood laws which allow great amount of predictability in 
physical processes or methods. Applicants methods go against his predictability in the disclosure does not provide adequate support or explanation as to how the claimed methods are achieved."

With respect to the "amount of direction provided by the inventor," the examiner found that

the specification discloses theories of the inventor and examples of using a light source to induce quantum entanglement but there is no direction as to how the light source actually generates a quantum entanglement. Applicant merely states that when the light source emits quantum entanglement generating members into a substance, they quantum-entangle with the substance. Does this mean that anytime someone or something is in the presence of a light source, it will be quantum entangled with other surrounding people and/or objects?

With respect to the "existence of working examples," the examiner found that "the disclosure is directed to applicant's theories and while there are examples provided (such as light sources which create quantum entanglement) these are not considered to be working examples because there is no evidence that the light sources another set ups for carrying out the invention are different than any other everyday light source such as a flashlight which will not function to generate quantum entanglement."

Finally, with respect to the "quantity of experimentation needed to make or use the invention" factor, the examiner found that "since applicant's claimed invention is directed to alternative physical phenomena that is not understood or accepted by mainstream physics, a great deal of undue experimentation would be required to carry out the invention."

The claims were also rejected under section 101 based on a lack of patentable utility. The examiner asserted that chemical substances must physically interact with the body in order to have an effect, and that "[i]f the substance is not physically administered within the body, the chemical and physical reactions and interactions required would not take place." Regarding the working examples, i.e., the "verification experiments," the examiner found it significant that the experiments were carried out on the inventor himself and friends and family members, and that the leftover prescriptions could have been expired and could been altered or tampered with in some way prior to the experiments. As such, the examiner found that the experiments were not sufficient to prove that quantum entanglement is the reason for the obtained results. "Merely theorizing that quantum entanglement may be responsible is not sufficient to establish a credible cause-and-effect relationship and is not sufficient evidence to conclude the method claims would produce these results."

As further evidence of inoperability, the examiner cited an article written for the Cosmic Log on MSNBC.com, summarizing an interview with theoretical physicist Lawrence Krauss, wherein Krauss states "it is true that quantum mechanics is extremely strange, and on extremely small scales for short times, all sorts of weird things happen." Krauss went on to say "we do, at subatomic scale, behave quantum mechanically. But we behave like classical objects for a reason: were big, we have lots of particles, they interact. All the weirdness of quantum mechanics gets washed out on the scale that we can experience. That's why we experience a classical world." Krauss further elaborated that: 
the weirdness of quantum mechanics is reserved for either very specially prepared configurations in the laboratory, or scales that are so small that quantum mechanical effects are significant... The quantum mechanical correlations ... that quantum mechanics brings up [are] true only for very specially prepared systems that are isolated from the rest the world completely. And we are certainly not isolated from the rest the world. We are bombarded by many things every second of the day, and as a result, we are not specially prepared quantum mechanical systems.

The examiner concluded by stating:

quantum entanglement between a patient's brain in a therapeutic substance cannot be created according to applicant's method. Humans, or even a brain alone, are not isolated systems and contain many billions of particles that wipe out any quantum mechanical effects that could theoretically be created if single particles of a system were isolated. Applicant's claimed method is not directed to isolated particles in a controlled environment, but rather to biological systems and everyday world interacting with one another; according to Krauss, this can only occur in a classical physics sense. Quantum mechanics and quantum interactions are not physically possible on a scale we experience.

In a response to the office action filed on June 10,2015, the applicant submitted two declarations under 37 CFR $\S 1.132$. The first declaration ("Declaration 1") included five peer-reviewed scientific publications by $\mathrm{Hu}$ describing the claimed technology and supporting experiments (much of the text of the patent specification appears to be taken directly from some of these publications). The declaration also includes copies of a UK patent and a Chinese Patent Certificate (accompanied by what is purportedly an official English translation) issued to the applicants and purportedly claiming the same or similar inventions to those disclosed in the pending U.S. application.

Notably, all five of the applicants' articles are published in one of two journals, NeuroQuantology or Progress in Physics. Wikipedia has this say about NeuroQuantology:

NeuroQuantology is a monthly peer-reviewed interdisciplinary scientific journal meant to cover the intersection of neuroscience and quantum mechanics. It was established in April 2003 and its subject matter almost immediately dismissed in The Lancet Neurology as "wild invention" and "claptrap". While the journal had a 2017 impact factor of 0.453 , ranking it 253rd out of 261 journals in the category "Neuroscience" as reported in the 2018 edition of Journal Citation Reports, Clarivate Analytics delisted the journal in its 2019 edition. . . . In the Norwegian Scientific Index, NeuroQuantology has been listed as "Level 0" since 2008, which means that it is not considered scientific and publications in the journal therefore do not fulfill the necessary criteria in order to count for public research funding. ${ }^{26}$

\footnotetext{
${ }^{26}$ https://en.wikipedia.org/wiki/NeuroQuantology (citing J. McCrone (2003). "Quantum mind". The Lancet Neurology. 2 (7): 450. doi:10.1016/s1474-4422(03)00466-6. PMID 12849132; "Journals Ranked by Impact: Neuroscience". 2017 Journal Citation Reports. Web of Science (Science ed.); Clarivate Analytics. 2018.
} 
Progress in Physics has been identified as a "fringe" scientific publication that "encourages pathological individualism" and "will consider all work without regard to affiliations."27 Nevertheless, "the papers it publishes have all the appearance of scientific papers." 28 Wikipedia categorizes Progress in Physics as a "fringe science journal."29

The UK patent, GB 2456987 B, does indeed appear to claim the use of quantum entanglement to remotely manipulate the temperature or $\mathrm{pH}$ of water. Claim 1, the only independent claim in the UK patent, recites:

A method of producing and measuring a non-local effect in a target substance through manipulating an originating substance which comprises the steps of:

selecting said target substance and said originating substance, said target substance being in a quantum entanglement with said originating substance;

positioning said target substance at a first location and said originating substance at a second location;

cooling, heating or adding a third substance to said originating substance; and

measuring with a high-precision instrument a change in weight, temperature and/or $\mathrm{pH}$ value of said target substance;

whereby said non-local effect is said change in weight, temperature and/or $\mathrm{pH}$ value of said target substance and said non-local effect is produced through a non-local process mediated by said quantum entanglement.

I do not have expertise in the patent law of the UK, but as far as I can tell from looking at the prosecution history of the UK patent, the examination never addressed any issue relating to credibility, operativeness, or utility. As a U.S. patent practitioner I find this somewhat surprising, and would be curious to hear what someone better versed in UK patent law thinks about the patent. Given the issues raised in the U.S. prosecution, I would think that the patent would be vulnerable to a challenge in the UK. But given the apparent inoperability of the claimed invention, there is no reason to think the patent will ever be of sufficient significance for someone to challenge it.

Neither do I have expertise in the patent law of China, and I cannot read Chinese, so I cannot independently verify what exactly was patented in China, but I have no reason to doubt Hu's

\footnotetext{
"Master Journal List". Intellectual Property \& Science. Clarivate Analytics. Retrieved 2019-05-29; and NeuroQuantology Norwegian Register for Scientific Journals, Series and Publishers).

${ }^{27}$ Harry Collins, Andrew Bartlett, and Luis Reyes-Galindo, Demarcating Fringe Science for Policy, 25 Perspectives in Science 411 (July 2017).

${ }^{28} I d$.

${ }^{29}$ Wikipedia, Category: Fringe science journals, available at https://en.wikipedia.org/wiki/Category:Fringe_sciencejournals.
} 
assertion that the subject matter covered by the Chinese patent is substantially the same as the subject matter for which the applicant sought patent protection in the U.S..

The second declaration filed by the applicants, ("Declaration 2"), contains 15 scientific articles by other scientists which, Hu argued, supported the operability of their inventions. In contrast with the "fringe" journals in which the applicants publish their work, a number of the articles presented in Declaration 2 are in highly respected mainstream journals, including Scientific American, Science, and Nature.

Along with the declarations, $\mathrm{Hu}$ (who represented themselves pro se) argued that the examiner had, "by only providing mere arbitrary and uninformed assertions of various 'wands factors', failed to satisfy the Office's burden to articulate a prima facie case of lack of enablement."

With regard to the examiner's assertion that "the invention appears to be drawn to theoretical physics," the applicant responded that "quantum entanglements at both the micro- and macroscopic scales have been experimentally proven to be real as evidenced by" the publications submitted in Declaration 2. Regarding the examiner's assertion that "the teachings of the prior art relevant to the instant invention are published an alternative physics journals associated with the inventor," the applicant faulted the examiner for allegedly providing no evidence of any association between the applicant and the alleged journal, and for failing to provide a factual basis for her assertion that the alleged journal is an "alternative physics journal." The applicant also disputed the examiner's assertion that the claimed invention is directed to alternative physical phenomena not understood or accepted by mainstream physics, arguing that quantum entanglement and non-local effects associated with quantum entanglement are not alternative physical phenomena but within the mainstream of science, as evidenced by the 15 publications by other scientists provided in Declaration 2.

In response to the rejection under section 101 for lack of utility, Hu complained:

$[\mathrm{H}] \mathrm{ere}$ is the Applicant's dilemma: the Examiner rejects just because she does not believe (or understand how) quantum entanglement can be created as claimed or persisted in the presence of the alleged decoherence and produce the experimentally observed non-local effect. By way of analogy, Bell invented telephone, disclose exactly how to build and use the telephone and further demonstrated that it actually works; but the examiner rejected because the examiner could not believe or did not understand that the electrical current could produce sound.

The examiner was not persuaded, and issued a final rejection, essentially reiterating the rationale of the first office action. The applicant filed a Request for Continued Examination (RCE), followed up by two more Rule 132 declarations offering up 10 more publications by other scientists which the applicant's asserted supported the operability of their invention.

\section{Hu's appeal}

When the examiner issued another final rejection, $\mathrm{Hu}$ appealed. On appeal, $\mathrm{Hu}$ presented the Board with essentially the same arguments made before the examiner, relying heavily on the 25 
publications by other scientists that were submitted in the form of declaration during the prosecution.

In the examiner's answer to the appeal brief, the examiner addressed the patents issued by China and the UK, as well as the applicants' peer-reviewed publications reporting the operability of the claimed invention, by noting that:

$[\mathrm{T}]$ he patentability of an invention in another country or publication of claimed subject matter any scientific journal is not material to patentability in the United States and the mere fact that the claimed subject matter was published or patented in another country does not provide evidence of a sufficient description, enablement of the claims, or credible utility. ${ }^{30}$

Regarding the experimental evidence provided in the specifications for the operability of the claimed invention, the examiner argued that "[e]merging and unpredictable technologies, which quantum entanglement is considered to be, require relatively more evidence to show possession of the invention. It is not sufficient to conclude that a certain effect, such as an increased heart rate, must have been caused by quantum entanglement absent any other evidence of quantum entanglement."

Regarding Hu's assertion that their test samples and testing environment are "in isolated in highly controlled laboratory conditions," the examiner disagreed, finding that " $[\mathrm{t}]$ he experiments outlined in the specification are performed on people (large biological systems) using substances and glassware container; these are not isolated environments of quantum entangling members such as electrons and photons acting on isolated particles or small groups of atoms."

The examiner emphasized that "the principle of quantum entanglement is not being disputed nor whether macroscopic systems may be entangled. [But even] if quantum entanglement can be created between two macroscopic objects or biological systems, this does not mean in the apparatus recited as generating quantum entanglement is automatically enabled."

On appeal, the Board affirmed the Examiner's rejections of the claims. The Board explained that:

we have no doubt that if Appellant's invention is able to use quantum entanglement to cause a therapeutic response in a subject by administering a pharmaceutical substance to water that is not actually consumed by that subject but was previously microwaved with water that is consumed by the subject it would be groundbreaking and revolutionary. However, due to the absence of any known scientific principles explaining how Appellants' invention could possibly operate in this manner, the absence of any cogent explanation in Appellant's specification regarding the general principals or mechanisms causing this to occur, and the absence of any verifiable test data reasonably attributable to the purported result, the Examiner reasonably characterized Appellants 'invention as being of an incredible nature.... There is no explanation offered as to why spin or any other quantum property of entangled particles would cause pharmacological changes in

\footnotetext{
${ }^{30}$ Ex Parte Huping Hu and Maoxin Wu., 2017 WL 11195640 (Patent Tr. \& App. Bd.).
} 
a discrete water sample only by virtue of having previously been microwaved with a water sample to which a pharmacological substances added. We are also not apprised of any data logically evincing such a pharmacological interaction has actually occurred. ${ }^{31}$

In In re Hu, the Federal Circuit affirmed. In an opinion authored by Judge Newman, the panel stated:

The Board did not err in requiring $\mathrm{Hu}$ to establish the operability of his asserted discoveries, in view of the conflict with ordinary experience as well as with established scientific principles. . . The PTO, as the nation's guardian of technologic invention, must be receptive to unusual concepts, for the core of invention is unobviousness. However, concepts that strain scientific principles are properly held to a heightened standard, typically measured by reproducibility of results. Here the Board was presented with an apparent departure from conventional scientific understanding, and the Board appropriately sustained the examiners' requirements for experimental verification. The Board applied a reasonable and objective standard, and acted reasonably in sustaining the examiners' requirements. Should further investigation bring peer recognition and verifiable results, the PTO and the scientific community would surely be interested. ${ }^{32}$

\section{Concluding thoughts}

Patents by their very nature can involve groundbreaking technologies, at times defying the expectations of experts in the field. Surprising and unexpected results that run counter to conventional wisdom are often pointed to as evidence refuting an allegation that a claimed invention is unpatentable for reasons of obviousness. And, indeed, some of the recent developments in quantum entanglement seem utterly fantastic for those of us whose intuition is grounded in the macroworld. Even mainstream physicists have apparently struggled in coming to terms with the implications of quantum entanglement, including its effects on macroscopic objects and living organisms. Nevertheless, it certainly seems that the PTO and Federal Circuit got it right in the case of $\mathrm{Hu}$ - if quantum entanglement of a sample of water on the scale of hundreds of milliliters could be so easily achieved with a microwave oven, it seems inconceivable that this fact would have eluded the many mainstream physicists working in the field.

One of the interesting aspects of In re Hu is the manner in which the purported inventors attempted to use their academic credentials, sworn affidavits, working examples, publications in peer-reviewed science journals, and patents issued in other jurisdictions as evidence of operativeness. Normally, such overwhelming objective evidence should win the day. The problem is, these are researchers and scientific publications on the fringe, rejected by the mainstream, and the PTO and Federal Circuit clearly understood this. As far as I know, there is

\footnotetext{
${ }^{31}$ Ex Parte Huping Hu \& Maoxin Wu, No. APPEAL 2018-003401, 2019 WL 2255476, at*5 (P.T.A.B. May 16, 2019).

322021 WL 1016437, at*9 (Fed. Cir. Mar. 17, 2021).
} 


\section{PRE-PRINT VERSION}

no explicit, formal doctrine of patent law that addresses the distinction between "fringe" and "mainstream" science, but in this case the PTO and court was able to recognize fringe science when they saw it. 